\title{
Clinical and prognostic significance of BRAF V600E mutation in non-metastatic cutaneous melanoma patients
}

\author{
F. TAS*, K. ERTURK \\ ${ }^{1}$ Department of Medical Oncology, Institute of Oncology, University of Istanbul, Istanbul, Turkey \\ *Correspondence: faruktas2002@yahoo.com
}

Received October 6, 2018 / Accepted January 30, 2019

\begin{abstract}
Because of the conflicting conclusions on BRAF mutations in the natural course of non-metastatic melanoma, their prognostic significance is still controversial. The present study aims to assess the prevalence and prognostic significance of BRAF V600E mutation and apprehend its association with clinicopathological features in stage I to III Turkish melanoma patients. A total of 93 adult stages I to III cutaneous primary melanoma patients were included in the study. BRAF V600E mutation was detected using the Real Time PCR. Median age was 52 years (range, 18 to 84 ) and $68.8 \%$ of the patients were males. Overall, BRAF V600E mutation was detected in 46.2\% (43/93) of the patients. In stages I and II, trunk was the most frequently affected localization $(47.1 \%)(\mathrm{p}=0.05)$ and regression was found more prevalent in BRAF-mutant patients (38.5\%) ( $\mathrm{p}=0.05)$. Furthermore, males were predominant among stage III BRAF-mutant patients $(80.8 \%)(\mathrm{p}=0.05)$, and both superficially spreading histology subtype $(45.0 \%)(\mathrm{p}=0.05)$ and lower mitotic rate $(36.4 \%)(\mathrm{p}=0.02)$ were also more commonly associated with stage III BRAF-mutant patients. A significantly favorable relapse free survival was found in stage III node-positive BRAF-mutant patients $(\mathrm{p}=0.02)$, on the other hand BRAF status was not found to be associated with relapse free survival in stage I and stage II patients $(\mathrm{p}=0.3)$. Moreover, there was no overall survival association between stages and BRAF status ( $\mathrm{p}=0.1$ for stage I-II and $\mathrm{p}=0.2$ for stage III). In conclusion, there is no prognostic value of BRAF V600E mutation on overall survival in stage I-III melanoma patients, yet its presence might indicate a decreased risk for development of relapse and/or metastasis in stage III melanoma patients.
\end{abstract}

Key words: melanoma, BRAF V600E, mutation, prognostic

BRAF, a proto-oncogene, belongs to the family of growth signal transduction RAF kinases and accounts for the regulation of the mitogen-activated protein kinases (MAPK) pathway that mediates cell division, differentiation and secretion [1]. A wide variety of BRAF mutations has been discovered in malignancies, most of which results in an amino acid substitution at position 600 in BRAF, from a valine to a glutamic acid (BRAF V600E) that eventually leads to activation of kinase pathway and cancer development $[1,2]$.

It is estimated that about $50 \%$ of skin melanomas harbor BRAF mutations $[1,2]$. BRAF V600E, the most common BRAF mutation in melanoma, results in increased kinase activity that leads to uncontrolled melanocyte cell growth, enhanced mitotic activity and melanoma tumor formation.

The prognostic significance of BRAF mutations in the natural course of melanoma is controversial [2-5]. Some studies showed poor survivals in metastatic BRAF-mutant patients [2-4], on the other hand others found no association between the tumor BRAF genotype and survival in metastatic settings [5]. Similar controversy exists for non-metastatic melanoma patients; some studies advocated [6] and some others negated [7-9] the assumption that BRAF mutation was a favorable prognostic factor in non-metastatic melanoma patients; however, there are also publications that found no association between BRAF-mutation and survival in such patients [10-15].

The prevalence of BRAF V600E mutation among Turkish melanoma patients has yet to be evaluated. The present study aims to assess the prevalence and prognostic significance of BRAF V600E mutation and apprehend its association with clinicopathological features in non-metastatic Turkish melanoma patients.

\section{Patients and methods}

Patients. A total of 93 adult Turkish Caucasian stage IIII primary skin melanoma patients with available information on the mutational status of the oncogene BRAF V600E 
were included in the analysis. Data were retrieved retrospectively from the charts of the patients who had been admitted, treated and followed up at Istanbul University Institute of Oncology, a single tertiary referral center. The disease was staged according to AJCC ( $7^{\text {th }}$ edition) staging system. Patients were treated and followed-up according to standard international guidelines including National Comprehensive Cancer Network guidelines.

BRAF V600E mutation was detected using the Real Time PCR in the formalin-fixed paraffin-embedded samples. Genomic DNA was extracted from melanoma cell positive paraffin embedded samples via the commercial kit Exgene FFPE Tissue DNA Kit (GeneAll Biotechnology Co, Seoul, Korea; Catalog no: 38-150) according to the kit's instructions. Fifty nanograms of DNA were then applied for BRAF V600E mutation analysis using a commercial kit with a detection sensitivity of $1 \%$ mutation load (BRAF Mutation Analysis Kit II, Entrogen, USA) in a ABI 7500 real time PCR machine (Applied Biosystems, USA) as described previously [16]. The allele-specific probe used to identify the V600E mutation was labeled with FAM. The study was reviewed and approved by our local ethics committee.

Statistical analysis. Comparisons between patient/disease variables and BRAF V600E mutation status were done using chi-square tests. Kaplan-Meier analysis was used for estimation of survival and differences in survivals were assessed by the log-rank statistics. Relapse-free survival (RFS) was calculated from the date of pathologic diagnosis to the date of clinical recurrence, which was defined as detected by imaging studies or by clinical examination. Overall survival (OS) was determined from the date of pathologic diagnosis to death resulting from any cause. A p value $\leq 0.05$ was considered significant. Statistical analysis was carried out using SPSS 21.0 software (SPSS Inc., Chicago, Illinois, USA).

\section{Results}

Patients. A total of 93 patients were included in this study. Median age was 52 years (range, 18 to 84 ) and $68.8 \%$ of the patients were males (Table 1$)$. The majority of patients $(n=54$, $58.1 \%)$ had stage III and the rest had stage I-II diseases at the initial diagnosis. Other histopathological and clinical features of the patients are presented in Table 1.

BRAF V600E mutation prevalence and its association with clinicopathological variables. Overall, BRAF V600E mutation was detected in $46.2 \%$ (43/93) of the patients (Table 1). Associations between BRAF V600E mutation and demographic, histopathological and clinical characteristics are shown in Table 1. The BRAF V600E status was strongly associated with gender; the prevalence was $79.1 \%$ and $20.9 \%$ in men and women, respectively $(\mathrm{p}=0.04)$. Trunk was the most frequently afflicted site in BRAF V600E-mutant patients (41.5\% vs. $28.6 \%$ ) whereas lower limbs were mostly affected in BRAF V600E-wild patients (46.9\% vs. $14.6 \%)$, $(\mathrm{p}=0.01)$. Furthermore, superficially spreading subtype was more frequently detected in BRAF V600E-mutant patients (51.6\% vs. $24.2 \%)$, on the other hand acral lentiginous type was more commonly associated with BRAF V600E-wild patients ( $30.3 \%$ vs. $6.5 \%),(\mathrm{p}=0.03)$. Additionally, regression was more prevalent in BRAF V600E-mutant patients (38.7\% vs. $13.8 \%),(\mathrm{p}=0.02)$. BRAF V600E mutation status was not found to be associated with other variables, such as age, Clark level, tumor thickness, ulceration, mitotic rate, lymph node status and stage of disease.

In stage I-II patients the mutation status was significantly associated with both the anatomical localization of the tumor and the presence of regression (Table 1). In BRAF-mutant patients of this group truncal lesions were predominant $(47.1 \%)(\mathrm{p}=0.05)$ and the majority of lesions harbored regression (38.5\%) ( $\mathrm{p}=0.05)$. Majority of BRAF-mutant patients in stage III group were males $(80.8 \%)(\mathrm{p}=0.05)$ and BRAFmutant lesions within this group of patients were found to harbor mostly superficially spreading histology subtype $(45.0 \%)(\mathrm{p}=0.05)$ and lower mitotic rate $(36.4 \%)(\mathrm{p}=0.02)$.

Disease progression. The overall relapse rate was $81.7 \%$ $(n=76)$ (Table 1). Local recurrence, i.e. recurrence in primary tumor site and in lymph node region, was seen in nearly half of the relapses (48.7\%), but distant metastases occurred either alone $(23.7 \%)$ or in co-existence with local diseases (27.6\%). No significant differences in recurrence rates were found, yet BRAF V600E-wild lesions compared to BRAF V600Emutant lesions had a tendency to relapse more frequently ( $88.0 \%$ vs. $74.4 \%$, respectively; $\mathrm{p}=0.09$ ). In stage I-II patients similar relapse rates between BRAF-mutant and BRAF-wild groups were observed $(\mathrm{p}=0.9)$, however in stage III patients relapse rate was significantly lower in BRAF V600E-mutant patients than BRAF V600E-wild patients $(69.2 \%$ vs. $92.9 \%$, respectively, $\mathrm{p}=0.02$ ).

The median time for any first relapse regarding the entire cohort was longer in the BRAF V600E-mutant group than the BRAF V600E-wild group (19.7 vs. 13.4 months, respectively, $\mathrm{p}=0.02$ ) (Table 2 and 3; Figure 1A). Moreover, similar significantly favorable relapse-free survival rate was observed in node positive patients ( $\mathrm{p}=0.02$ ); however, stage I-II patients had no survival differences between BRAF V600E-statuses $(\mathrm{p}=0.3)$ (Table 2 and Figure $1 \mathrm{~B}$ and $1 \mathrm{C})$.

Survival. Albeit not statistically significant, BRAF V600Emutant patients tended to have a longer median survival time compared to BRAF V600E-wild patients (28.9 vs. 22.4 months, respectively; $\mathrm{p}=0.09$ ) (Table 2 and 3; Figure 2A). Furthermore, no overall survival differences between BRAF V600E statuses were observed in stages, i.e. stage I-II and stage III ( $\mathrm{p}=0.1$ and $\mathrm{p}=0.2$ ) (Table 2 and Figure $2 \mathrm{~B}$ and $2 \mathrm{C}$ ).

\section{Discussion}

In the present study, we investigated the prevalence of BRAF V600E mutation in 93 non-metastatic Turkish melanoma patients and found that it existed in $46.2 \%$ of lesions; that is the similar prevalence ratio as determined 
Table 1. Patient and disease characteristics according to BRAF V600E mutation status and clinical stage.

\begin{tabular}{|c|c|c|c|c|c|c|c|c|c|c|}
\hline \multirow[b]{2}{*}{ Variable } & \multicolumn{4}{|c|}{ All patients } & \multicolumn{3}{|c|}{ Stage I-II } & \multicolumn{3}{|c|}{ Stage III } \\
\hline & n (\%) & $\begin{array}{c}\text { BRAF(-) } \\
\text { n (\%) }\end{array}$ & $\begin{array}{c}\text { BRAF(+) } \\
\text { n (\%) }\end{array}$ & p-value & $\begin{array}{c}\text { BRAF(-) } \\
\text { n (\%) }\end{array}$ & $\begin{array}{c}\text { BRAF(+) } \\
\text { n (\%) }\end{array}$ & p-value & $\begin{array}{c}\text { BRAF(-) } \\
\text { n (\%) }\end{array}$ & $\begin{array}{c}\text { BRAF(+) } \\
\text { n (\%) }\end{array}$ & p-value \\
\hline \multicolumn{11}{|l|}{ Age, years } \\
\hline$<35$ & $16(17.2)$ & $6(12.0)$ & $10(23.3)$ & \multirow{4}{*}{0.2} & $2(9.1)$ & $4(23.5)$ & \multirow{4}{*}{0.2} & $4(14.3)$ & $6(23.1)$ & \multirow{4}{*}{0.7} \\
\hline $35-49$ & $22(23.7)$ & $12(24.0)$ & $10(23.3)$ & & $6(27.3)$ & $4(23.5)$ & & $6(21.4)$ & $6(23.1)$ & \\
\hline $50-69$ & $42(45.2)$ & $22(44.0)$ & $20(46.5)$ & & $10(45.5)$ & $9(52.9)$ & & $12(42.9)$ & $11(42.3)$ & \\
\hline$\geq 70$ & $13(14.0)$ & $10(20.0)$ & $3(7.0)$ & & $4(18.2)$ & $0(0.0)$ & & $6(21.4)$ & $3(11.5)$ & \\
\hline \multicolumn{11}{|l|}{ Sex } \\
\hline Female & $29(31.2)$ & $20(40.0)$ & $9(20.9)$ & \multirow[t]{2}{*}{0.04} & $8(36.4)$ & $4(23.5)$ & \multirow[t]{2}{*}{0.3} & $12(42.9)$ & $5(19.2)$ & 0.05 \\
\hline Male & $64(68.8)$ & $30(60.0)$ & $34(79.1)$ & & $14(63.6)$ & $13(76.5)$ & & $16(57.1)$ & $21(80.8)$ & \\
\hline Site of lesion & & & & & & & & & & \\
\hline Head\&neck & $16(17.8)$ & $7(14.3)$ & $9(22.0)$ & & $5(22.7)$ & $6(35.3)$ & & $2(7.4)$ & $3(12.5)$ & \\
\hline Trunk & $31(34.4)$ & $14(28.6)$ & $17(41.5)$ & 0.01 & $6(27.3)$ & $8(47.1)$ & 0.05 & $8(29.6)$ & $9(37.5)$ & 0.2 \\
\hline Upper limb & $14(15.6)$ & $5(10.2)$ & $9(22.0)$ & & $1(4.5)$ & $2(11.8)$ & & $4(14.8)$ & $7(29.2)$ & \\
\hline Lower limb & $29(32.2)$ & $23(46.9)$ & $6(14.6)$ & & $10(45.5)$ & $1(5.9)$ & & $13(48.1)$ & $5(20.8)$ & \\
\hline Histopathology & & & & & & & & & & \\
\hline SSM & $24(37.5)$ & $8(24.2)$ & $16(51.6)$ & & $6(35.3)$ & $7(63.6)$ & & $2(12.5)$ & $9(45.0)$ & \\
\hline NM & $27(42.2)$ & $14(42.4)$ & $13(41.9)$ & 0.03 & $5(29.4)$ & $4(36.4)$ & 0.1 & $9(56.2)$ & $9(45.0)$ & 0.05 \\
\hline ALM & $12(18.8)$ & $10(30.3)$ & $2(6.5)$ & & $5(29.4)$ & $0(0.0)$ & & $5(31.2)$ & $2(10.0)$ & \\
\hline LMM & $1(1.16)$ & $1(3.0)$ & $0(0.0)$ & & $1(5.9)$ & $0(0.0)$ & & $0(0.0)$ & $0(0.0)$ & \\
\hline Clark level & & & & & & & & & & \\
\hline I-III & $13(17.8)$ & $5(13.5)$ & $8(22.2)$ & 0.3 & $5(27.8)$ & $6(42.9)$ & 0.3 & $0(0.0)$ & $2(9.1)$ & 0.1 \\
\hline IV-V & $60(82.2)$ & $32(86.5)$ & $28(77.8)$ & & $13(72.2)$ & $8(57.1)$ & & $19(100)$ & $20(90.9)$ & \\
\hline Breslow depth & & & & & & & & & & \\
\hline $\mathrm{T} 1$ & $2(2.7)$ & $2(5.3)$ & $0(0.0)$ & & $2(11.1)$ & $0(0.0)$ & & $0(0.0)$ & $0(0.0)$ & \\
\hline $\mathrm{T} 2$ & $8(10.7)$ & $3(7.9)$ & $5(13.5)$ & 0.2 & $2(11.1)$ & $4(28.6)$ & 0.2 & $1(5.0)$ & $1(4.3)$ & 0.5 \\
\hline T3 & $31(41.3)$ & $19(50.0)$ & $12(32.4)$ & & $10(55.6)$ & $5(35.7)$ & & $9(45.0)$ & $7(30.4)$ & \\
\hline $\mathrm{T} 4$ & $34(45.3)$ & $14(36.8)$ & $20(54.1)$ & & $4(22.2)$ & $5(35.7)$ & & $10(50.0)$ & $15(65.2)$ & \\
\hline TIL & & & & & & & & & & \\
\hline No & $43(64.2)$ & $24(70.6)$ & $19(57.6)$ & 0.2 & $11(73.3)$ & $5(41.7)$ & 0.09 & $13(68.4)$ & $14(66.7)$ & 0.9 \\
\hline Yes & $24(35.8)$ & $10(29.4)$ & $14(42.4)$ & & $4(26.7)$ & $7(58.3)$ & & $6(31.6)$ & $7(33.3)$ & \\
\hline Mitotic rate, $/ \mathrm{m}$ & & & & & & & & & & \\
\hline$\leq 2$ & $16(23.9)$ & $6(18.2)$ & $10(29.4)$ & 0.2 & $5(31.2)$ & $2(16.7)$ & 0.3 & $1(5.9)$ & $8(36.4)$ & 0.02 \\
\hline$>2$ & $51(76.1)$ & $27(81.8)$ & $24(70.6)$ & & $11(68.8)$ & $10(83.3)$ & & $16(94.1)$ & $14(63.6)$ & \\
\hline Ulceration & & & & & & & & & & \\
\hline No & $21(30.0)$ & $10(28.6)$ & $11(31.4)$ & 0.8 & $6(37.5)$ & $3(23.1)$ & 0.4 & $4(21.1)$ & $8(36.4)$ & 0.2 \\
\hline Yes & $49(70.0)$ & $25(71.4)$ & $24(68.6)$ & & $10(62.5)$ & $10(76.9)$ & & $15(78.9)$ & $14(63.6)$ & \\
\hline Regression & & & & & & & & & & \\
\hline No & $44(73.3)$ & $25(86.2)$ & $19(61.3)$ & 0.02 & $13(92.9)$ & $8(61.5)$ & 0.05 & $12(80.0)$ & $11(61.1)$ & 0.2 \\
\hline Yes & $16(26.7)$ & $4(13.8)$ & $12(38.7)$ & & $1(7.1)$ & $5(38.5)$ & & $3(20.0)$ & 7 (38.9) & \\
\hline Neurotropism & & & & & & & & & & \\
\hline No & $37(94.9)$ & $13(92.9)$ & $24(96.0)$ & 0.6 & $6(85.7)$ & $10(100)$ & 0.2 & $7(100)$ & $14(93.3)$ & 0.4 \\
\hline Yes & $2(5.1)$ & $1(7.1)$ & $1(4.0)$ & & $1(14.3)$ & $0(0.0)$ & & $0(0.0)$ & $1(6.7)$ & \\
\hline LVI & & & & & & & & & & \\
\hline No & $54(84.4)$ & $25(86.2)$ & $29(82.9)$ & 0.7 & $13(92.9)$ & $13(100)$ & 0.3 & $12(80.0)$ & $16(72.7)$ & 0.6 \\
\hline Yes & $10(15.6)$ & $4(13.8)$ & $6(17.1)$ & & $1(7.1)$ & $0(0.0)$ & & $3(20.0)$ & $6(27.3)$ & \\
\hline Node stage & & & & & & & & & & \\
\hline N1 & $22(40.7)$ & $13(46.4)$ & $9(34.6)$ & & & & & $13(46.4)$ & $9(34.6)$ & \\
\hline $\mathrm{N} 2$ & $20(37.0)$ & $9(32.1)$ & $11(42.3)$ & 0.6 & - & - & - & $9(32.1)$ & $11(42.3)$ & 0.6 \\
\hline N3 & $12(22.2)$ & $6(21.4)$ & $6(23.1)$ & & & & & $6(21.4)$ & $6(23.1)$ & \\
\hline Stage & & & & & & & & & & \\
\hline I-II & 39 (41.9) & $22(44.0)$ & $17(39.5)$ & 0.6 & - & - & - & - & - & - \\
\hline III & $54(58.1)$ & $28(56.0)$ & $26(60.5)$ & & & & & & & \\
\hline Adjuvant therap & & & & & & & & & & \\
\hline No & $18(33.3)$ & $8(28.6)$ & $10(38.5)$ & 0.4 & - & - & - & $8(28.6)$ & $10(38.5)$ & 0.4 \\
\hline Yes & $36(66.7)$ & $20(71.4)$ & $16(61.5)$ & & & & & $20(71.4)$ & $16(61.5)$ & \\
\hline Relapse & & & & & & & & & & \\
\hline No & $17(18.3)$ & $6(12.0)$ & $11(25.6)$ & 0.09 & $4(18.2)$ & $3(17.6)$ & 0.9 & $2(7.1)$ & $8(30.8)$ & 0.02 \\
\hline Yes & $76(81.7)$ & $44(88.0)$ & $32(74.4)$ & & $18(81.8)$ & $14(82.4)$ & & $26(92.9)$ & $18(69.2)$ & \\
\hline Relapse site & & & & & & & & & & \\
\hline Local only & $37(48.7)$ & $19(43.2)$ & $18(56.2)$ & & $10(55.6)$ & $7(50.0)$ & & $9(34.6)$ & $11(61.1)$ & \\
\hline Mixed & $21(27.6)$ & $13(29.5)$ & $8(25.0)$ & 0.5 & $5(27.8)$ & $5(35.7)$ & 0.9 & $8(30.8)$ & $3(16.7)$ & 0.2 \\
\hline Distant only & $18(23.7)$ & $12(27.3)$ & $6(18.8)$ & & $3(16.7)$ & $2(14.3)$ & & $9(34.6)$ & $4(22.2)$ & \\
\hline Last status & & & & & & & & & & \\
\hline Dead & $43(46.2)$ & $27(54.0)$ & $16(37.2)$ & 0.1 & $9(40.9)$ & $3(17.6)$ & 0.1 & $18(64.3)$ & $13(50.0)$ & 0.2 \\
\hline Alive & $50(53.8)$ & $23(46.0)$ & $27(62.8)$ & & $13(59.1)$ & $14(82.4)$ & & $10(35.7)$ & $13(50.0)$ & \\
\hline
\end{tabular}


in other publications [2]. We also identified that BRAF V600E mutation was associated with some of the prognostic variables, such as sex, anatomical localization of the lesion and histopathological features. The majority of BRAF V600E-mutant patients were men and most of the lesions were truncal. BRAF-mutant lesions were commonly found in association with superficially spreading subtype and frequently harbored tumor regression. Our findings concur with the previously published data [6-15]. We found that BRAF V600E mutation had no prognostic impact on overall survival for either the entire cohort or the individual stages. However, there was a significant association between the presence of BRAF V600E mutation and decrease in recurrence/metastasis for the entire cohort and stage III patients.

In literature, controversy exists for the prognostic role of BRAF V600 in non-metastatic cutaneous melanoma patients [6-15]. Some data support that BRAF V600 mutation is an independent favorable prognostic factor [6]. In a recent

Table 2. Survival rates of patients.

\begin{tabular}{lccccc}
\hline \multirow{2}{*}{ Patients } & \multicolumn{2}{c}{ Relapse-free survival } & & \multicolumn{2}{c}{ Overall survival } \\
\cline { 2 - 3 } \cline { 5 - 6 } & Median (range) & p-value & & Median (range) & p-value \\
\hline All patients & & & & & \\
BRAF(-) & $13.4(0.7-76.8)$ & 0.02 & & $22.4(0.7-109.1)$ & 0.09 \\
BRAF(+) & $19.7(1.4-162.8)$ & & & $28.9(1.4-166.9)$ & \\
Stage I-II & & & & & \\
BRAF(-) & $21.6(0.7-76.8)$ & 0.3 & & $38.3(0.7-109.1)$ & 0.1 \\
BRAF(+) & $25.1(1.4-162.8)$ & & & $38.3(1.4-166.9)$ & \\
Stage III & & & & \\
BRAF(-) & $12.4(3.4-44.7)$ & 0.02 & & $20.2(4.0-100.0)$ & 0.2 \\
BRAF(+) & $15.8(3.1-94.9)$ & & & $23.0(4.9-127.6)$ & \\
\hline
\end{tabular}

German trial on 217 melanoma patients (most of them (96\%) were stage I-III patients) BRAF mutation was identified in $40.1 \%$ of the samples [6]. BRAF mutation was significantly associated with younger age at initial diagnosis, less mitotic activity, rarely detected acral lentiginous histological subtype and truncal lesion. BRAF-mutant melanoma individuals showed a tendency for better overall survival and the time to progression in these patients was longer. On the other hand, there are also studies with negative observations, i.e. advocating that BRAF mutation portends poor prognosis [7-9]. An Australian study on 79 stage III melanoma patients revealed that $40.5 \%$ of lesions harbored BRAF mutation and its presence predicted poor outcome [7]. Furthermore, Picard et al. also obtained at least one BRAF mutation in 32 of the 72 patients with a positive sentinel lymph node [8]. The only clinical feature associated with BRAF status was metastatic burden of the involved lymph nodes and they observed that BRAF mutation was an indicator of poor prognosis in these patients.

However, there are many other trials that have reported no correlation between BRAF mutation and prognosis [10-15]. In another German study, the prognostic impact of V600 tumor mutation in 437 stage III melanoma patients was investigated [10]. Mutation was detected in $38.7 \%$ of lesions and associated with age, histopathological subtype and mitotic rate. There was no difference in overall survival, but distant metastasis free survival was found poor in BRAF mutant patients. Another study on 223 melanoma patients showed that $48.9 \%$ of the lesions were BRAF-mutant and they were associated with aggressive tumor features [11]. Patients with mutation presented with more advanced clinical stages; they tended to have stage III disease $(n=52)$ at diagnosis and demonstrated the highest rate of ulceration.

Table 3. Univariate analyses of variables associated with relapse-free survival (RFS) and overall survival (OS).

\begin{tabular}{|c|c|c|c|c|c|c|}
\hline \multirow{2}{*}{ Variables } & \multicolumn{3}{|c|}{ RFS } & \multicolumn{3}{|c|}{ OS } \\
\hline & HR & 95\%CI & p-value & HR & 95\%CI & p-value \\
\hline Age & 1.122 & $0.702-1.794$ & 0.6 & 0.630 & $0.345-1.149$ & 0.1 \\
\hline Sex & 0.875 & $0.526-1.458$ & 0.6 & 0.942 & $0.480-1.849$ & 0.8 \\
\hline Site of lesion & 1.009 & $0.637-1.599$ & 0.9 & 0.948 & $0.518-1.735$ & 0.8 \\
\hline Histopathology & 1.238 & $0.681-2.250$ & 0.4 & 1.277 & $0.607-2.684$ & 0.5 \\
\hline Clark invasion & 1.164 & $0.598-2.263$ & 0.6 & 1.676 & $0.588-4.777$ & 0.3 \\
\hline Breslow depth & 1.182 & $0.576-2.425$ & 0.6 & 1.483 & $0.520-4.277$ & 0.4 \\
\hline Ulceration & 1.636 & $0.906-2.955$ & 0.1 & 1.458 & $0.686-3.102$ & 0.3 \\
\hline Mitotic rate & 1.950 & $1.030-3.890$ & 0.05 & 1.311 & $0.591-2.908$ & 0.5 \\
\hline Lymphovascular invasion & 2.139 & $0.931-4.913$ & 0.07 & 2.404 & $0.893-6.473$ & 0.08 \\
\hline Neurotropism & 0.045 & $0.000-1034$ & 0.5 & 0.046 & $0.000-2109$ & 0.8 \\
\hline Tumor infiltrating lymphocytes & 1.011 & $0.571-1.792$ & 0.9 & 1.002 & $0.480-2.092$ & 0.9 \\
\hline Regression & 1.045 & $0.535-2.042$ & 0.8 & 1.484 & $0.646-3.410$ & 0.3 \\
\hline Stage of disease & 0.914 & $0.866-0.965$ & 0.001 & 0.888 & $0.824-0.957$ & 0.002 \\
\hline Adjuvant therapy & 1.648 & $0.868-3.126$ & 0.1 & 1.396 & $0.649-3.003$ & 0.3 \\
\hline Relapse & - & - & - & 0.279 & $0.099-0.786$ & 0.01 \\
\hline BRAF(V600E) mutation & 0.584 & $0.363-0.941$ & 0.02 & 0.575 & $0.304-1.090$ & 0.09 \\
\hline
\end{tabular}



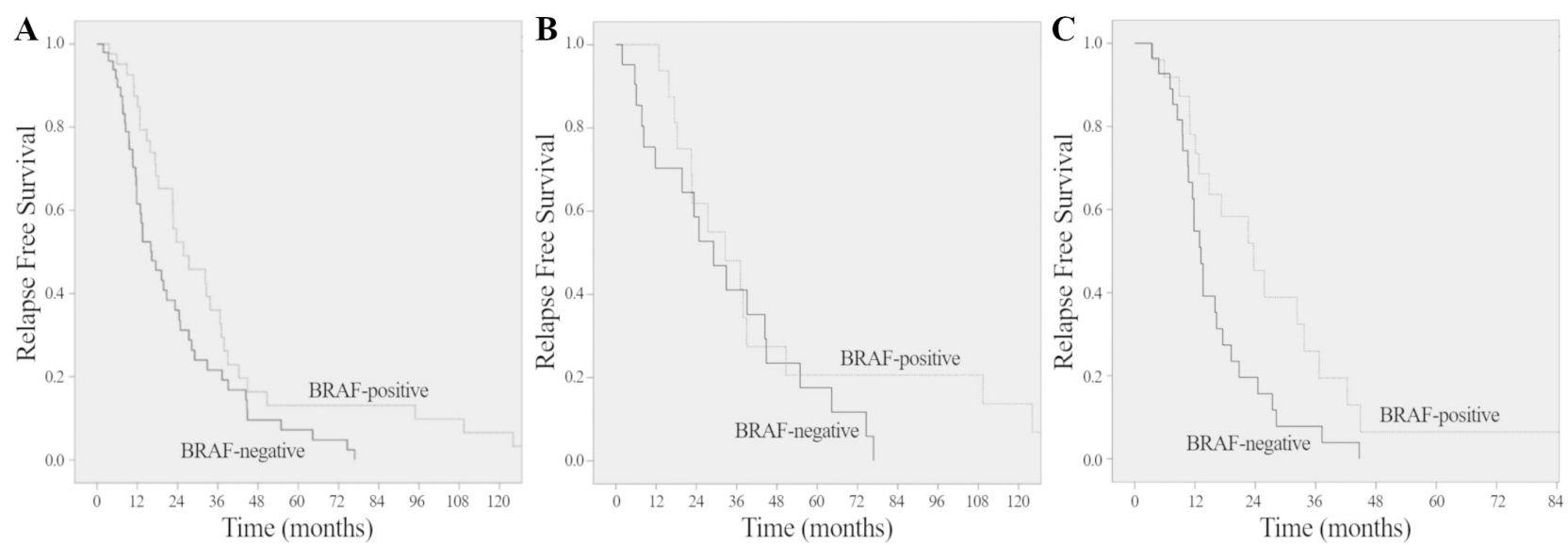

Figure 1. Relapse-free survival curves of A) all patients according to BRAF V600E mutation ( $p=0.02)$, B) stage I-II patients according to BRAF V600E mutation $(\mathrm{p}=0.3)$ and $\mathrm{C})$ stage III patients according to BRAF V600E mutation $(\mathrm{p}=0.02)$.
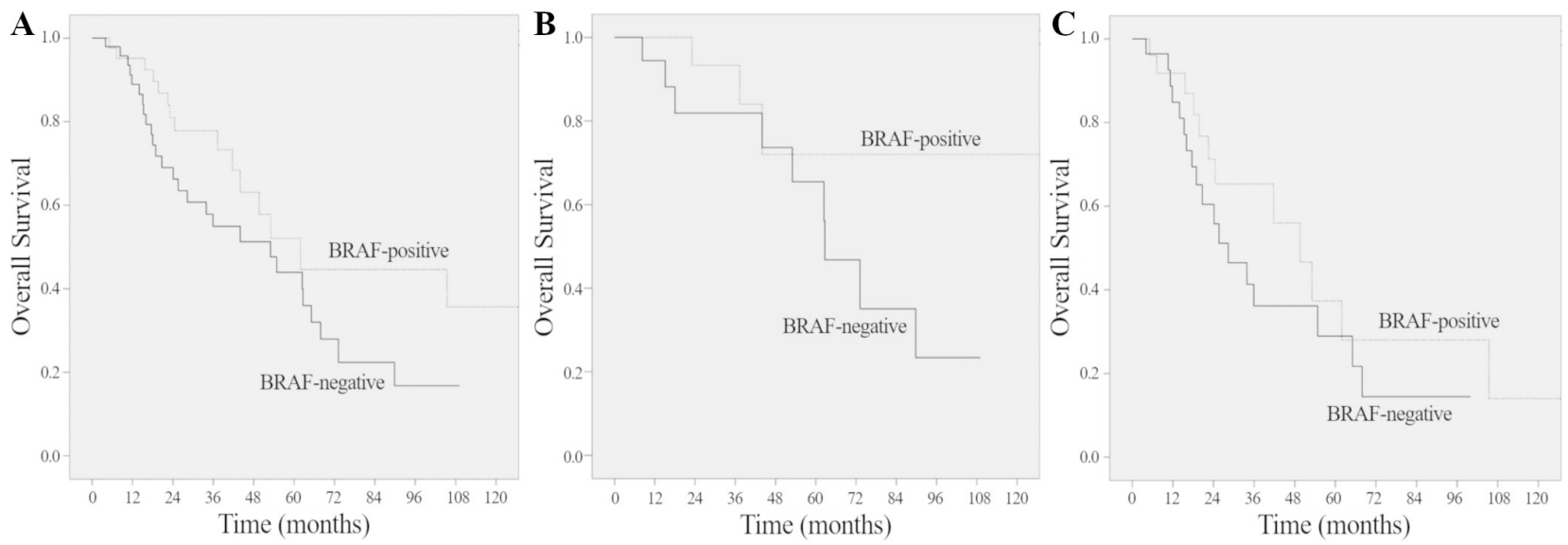

Figure 2. Overall survival curves of $A$ ) all patients according to BRAF V600E mutation ( $p=0.09)$, B) stage I-II patients according to BRAF V600E mutation $(\mathrm{p}=0.1)$ and $\mathrm{C})$ stage III patients according to BRAF V600E mutation $(\mathrm{p}=0.2)$.

In spite of this, no difference in survival among the stage III patients was found when stratified according to the presence or absence of mutation. Additionally, in a study of a group of seventy-seven primary skin melanoma patients (majority, $\mathrm{n}=66$, had non-metastatic stage) from a cancer reference center in Brazil no statistically significant association was found between the presence of the BRAF V600E mutation and clinical or prognostic parameters [15].

In conclusion, our findings are agreement with other trials, which investigated the frequency of BRAF V600E mutation and associations between the mutation and clinicopathological features in non-metastatic melanoma patients. Even though it has no prognostic impact on overall survival in stage I-III melanoma patients BRAF V600E mutation might indicate a reduced risk for relapse and/or metastasis in stage III patients. The potential role of the BRAF V600E mutation status as a prognostic indicator in non-metastatic melanoma patients needs to be investigated in larger studies.
Acknowledgements: We thank Prof. Ugur Gezer for valuable contribution in the preparation of the manuscript.

\section{References}

[1] BHATIA P, FRIEDLANDER P, ZAKARIA EA, KANDIL E. Impact of BRAF mutation status in the prognosis of cutaneous melanoma: an area of ongoing research. Ann Transl Med 2015; 3: 24. https://doi.org/10.3978/j.issn.23055839.2014.12.05

[2] SAFAEE ARDEKANI GS, JAFARNEJAD SM, TAN L, SAEEDI A, LI G. The prognostic value of BRAF mutation in colorectal cancer and melanoma: a systemic review and meta-analysis. PLoS One 2012; 7: e47054. https://doi. org/10.1371/journal.pone.0047054

[3] MENZIES AM, HAYDU LE, VISINTIN L, CARLINO MS, HOWLE JR et al. Distinguishing clinicopathologic features of patients with V600E and V600K BRAF-mutant metastatic melanoma. Clin Cancer Res 2012; 18: 3242-3249. https:// doi.org/10.1158/1078-0432.CCR-12-0052 
[4] LONG GV, MENZIES AM, NAGRIAL AM, HAYDU LE, HAMILTON AL et al. Prognostic and clinicopathologic associations of oncogenic BRAF in metastatic melanoma. J Clin Oncol 2011; 29: 1239-1246. https://doi.org/10.1200/ JCO.2010.32.4327

[5] EDLUNCH-ROSE E, EGYHAZI S, OMHOLT K, MANSSON-BRAHME E, PLATZ E et al. NRAS and BRAF mutations in melanoma tumors in relation to clinical characteristics: a study based on mutation screening by pyrosequencing. Melanoma Res 2006; 16: 471-478. https://doi. org/10.1097/01.cmr.0000232300.22032.86

[6] HEPPT MV, SIEPMANN T, ENGEL J, SCHUBERTFRITSCHLE G, ECKEL R et al. Prognostic significance of BRAF and NRAS mutations in melanoma: a German study from routine care. BMC Cancer 2017; 17: 536. https://doi. org/10.1186/s12885-017-3529-5

[7] MANN GJ, PUPO GM, CAMPAIN AE, CARTER CD, SCHRAMM SJ et al. BRAF mutation, NRAS mutation, and the absence of an immune-related expressed gene profile predict poor outcome in patients with stage III melanoma. J Invest Dermatol 2013; 133: 509-517. https://doi.org/10.1038/ jid.2012.283

[8] PICARD M, DANG NP, D'INCAN M, MANSARD S, DECHELOTTE $P$ et al. Is BRAF a prognostic factor in stage III skin melanoma? A retrospective study of 72 patients after positive sentinel lymph node dissection. Br J Dermatol 2014; 171: 108-114. https://doi.org/10.1111/bjd.12939

[9] MOREAU S, SAIAG P, AEGERTER P, BOSSET D, LONGVERT C et al. Prognostic value of BRAF V600 mutations in melanoma patients after resection of metastatic lymph nodes. Ann Surg Oncol 2012; 19: 4314-4321. https://doi. org/10.1245/s10434-012-2457-5
[10] MECKBACH D, BAUER J, PFLUGFELDER A, MEIER F, BUSCH C et al. Survival according to BRAF-V600 tumor mutation - an analysis of 437 patients with primary melanoma. PLoS One 2014; 9: e86194. https://doi.org/10.1371/ journal.pone.0086194

[11] ELLERHORST J, GREENE VR, EKMEKCIOGLU S, WARNEKE CL, JOHNSON MM et al. Clinical correlates of NRAS and BRAF mutations in primary human melanoma. Clin Cancer Res 2011; 17: 229-235. https://doi. org/10.1158/1078-0432.CCR-10-2276

[12] MALDONADO JL, FRIDLYAND J, PATEL H, JAIN AN, BUSAM K et al. Determinants of RAF mutations in primary melanomas. J Natl Cancer Inst 2003; 95: 1878-1890.

[13] SHINOZAKI M, FUJIMOTO A, MORTON DL, HOON DS. Incidence of BRAF oncogene mutation and clinical relevance for primary cutaneous melanomas. Clin Cancer Res 2004; 10: 1753-1757. https://doi.org/10.1158/1078-0432. CCR-1169-3

[14] AKSLEN LA, ANGELINI S, STRAUME O, BACHMANN IM, MOLVEN A et al. BRAF and NRAS mutations are frequent in nodular melanoma but are not associated with tumor cell proliferation or patient survival. J Invest Dermatol 2005; 125: 312-317. https://doi.org/10.1111/j.0022202X.2005.23788.x

[15] INUMARU JS, GORDO KI, FRAGA JUNIOR AC, SILVA AM, LEAL CB et al. Analysis of the BRAF V600E mutation in primary cutaneous melanoma. Gen Mol Res 2014; 13: 2840-2848. https://doi.org/10.4238/2014.January.22.8

[16] KIMBRELL HZ, SHOLL AB, RATNAYAKA S, JAPA S, LACEY $M$ et al. BRAF testing in multifocal papillary thyroid carcinoma. Biomed Res Int 2015; 2015: 486391. https://doi. org/10.1155/2015/486391 